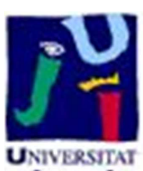

Título artículo / Títol article: Maturity Model for Interoperability Potential Measuremen

Autores / Autors

Cristina Camposa, Ricardo Chalmeta, Reyes Grangela, Raúl Poler

Revista:

Information Systems Management

Versión / Versió:

Postprint del autor

Cita bibliográfica / Cita bibliogràfica (ISO 690):
CAMPOS, Cristina, et al. Maturity Model for Interoperability Potential Measurement. Information Systems

Management, 2013, vol. 30, no 3, p. 218-234.

url Repositori UJI: 


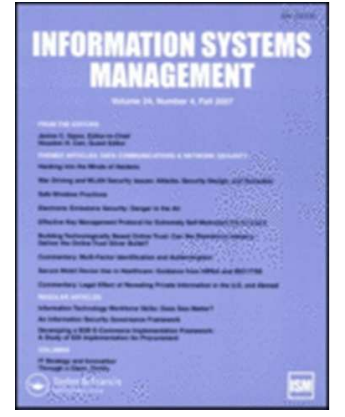

\section{MATURITY MODEL FOR INTEROPERABILITY POTENTIAL MEASUREMENT}

\begin{tabular}{|r|l|}
\hline Journal: & Information Systems Management \\
\hline Manuscript ID: & UISM-2011-027.R1 \\
\hline Manuscript Type: & Original Manuscript \\
\hline Keywords: & $\begin{array}{l}\text { Alignment of IS Organization, E-Government, Enterprise Systems, Inter- } \\
\text { organizational information systems }\end{array}$ \\
\hline \multicolumn{2}{|l}{} \\
\hline
\end{tabular}

SCHOLARONE ${ }^{\mathrm{m}}$

Manuscripts

URL: http:/mc.manuscriptcentral.com/uism Email: janice.sipior@villanova.edu 


\title{
MATURITY MODEL FOR INTEROPERABILITY POTENTIAL
}

\section{MEASUREMENT}

\begin{abstract}
Interoperability Potential concerns the level of preparation of an enterprise to establish an efficient collaboration with possible partners. In order to improve their interoperability, enterprises need to know what level of maturity they have achieved. This paper proposes a complete maturity model consisting in a methodology and a reference set of parameters with which to measure interoperability potential. In order to clarify the proposal an example of its application to a real case is described.
\end{abstract}

\section{KEYWORDS}

Interoperability Measurement; Interoperability Potential; Measurement Parameters; Maturity Model; Methodology

\section{INTRODUCTION}

In the current economic context enterprises must collaborate with one another efficiently in order to minimize costs, offer new services, deal with new challenges, and be more competitive both, in times of crisis or economic recession, and in times of growth.

One of the main problems that enterprises face when it comes to establishing efficient collaborative working relationships is the lack of cultural, conceptual, organizational, process and technological compatibility (Doumeingts \& Chen, 2003). 
The concept of enterprise interoperability thus appears as a solution to such problems. Many definitions of interoperability have been put forward over the years (Ford, Colombi, Graham, \& Jacques, 2007). Nevertheless, in this paper enterprise interoperability is defined as the capacity that enterprises and organizations have to collaborate in an efficient manner while preserving their own identities and their own ways of doing business through mechanisms that act as facilitators. In this context, preserving their identity means that the enterprise does not substantially modify its structure and processes in order to achieve compatibility with other enterprises.

Interoperability is considered to be achieved if the efficient collaboration takes place, at least, in the business, knowledge and information and communication technologies (ICT) layers, and also considering semantics aspects, as a transversal layer, that complement the previous three ones (Chen, Vallespir, \& Daclin, 2008).

To improve enterprise interoperability, it is necessary to analyze the current situation and perform a diagnosis of it so as to be able to identify any problems that might exist as well as opportunities for improvement. Within this process of evaluation of enterprise interoperability, different metrics have to be defined in order to assess the aspects that favor or restrict it (Chen \& Daclin, 2007). In addition, these metrics must be organized in a maturity model. A maturity model defines the states or levels at which an enterprise or system can be situated, a set of good practices, goals and quantifiable parameters that make it possible to determine on which of the levels the enterprise currently stands, and also a series of proposals with which to evolve from one level of maturity to a higher one (Ahern, Clouse, \& Turner, 2004). The concept of maturity models is not restricted exclusively to the field of enterprise interoperability, but is actually used in practice to measure the extent to which any technology or system is being adopted in the enterprise. Different types of 
interoperability can be measured by maturity models. In this paper we focused on interoperability potential measurement, which concerns the capability of an enterprise to interoperate with an unknown partner.

Although the literature offers different approaches to maturity models for measuring enterprise interoperability, these proposals focus on justifying the importance of measuring interoperability and on defining the possible levels an enterprise may find itself on, depending on its capacity to interoperate (Chen \& Daclin, 2007). Yet, none of the proposals define (1) what to measure, that is, they do not state what parameters are suitable for analyzing or evaluating enterprise interoperability, or (2) how to measure, that is to say, they do not propose appropriate methods for obtaining those parameters and evaluating them (which would enable them to determine the level of interoperability achieved by an enterprise or a process) in a satisfactory way. Thus, existing interoperability maturity models offer a good starting point, but must be improved. To solve this problem, this paper describes a potential interoperability Maturity Model that structures the measuring process in different views, identifies the parameters to be measured in each view, and proposes a detailed methodology to offer guidance in the process of measuring and evaluating those parameters. In order to validate and to evaluate the applicability and benefits of this proposal, a description of the use of the model to an enterprise in the textile sector is shown.

This paper is organized as follows: section 2 reviews the approaches and the projects carried out on maturity models that were considered during the development of the proposal; section 3 consists of the maturity model proposed, that includes the measurement parameters, the methodology and its phases; section 4 outlines how the framework was applied to a real enterprise and discusses practical aspects of this application. Finally, in section 5, conclusions and future work are showed. 


\section{LITERATURE REVIEW IN INTEROPERABILITY AND MATURITY MODELS}

In the context of enterprise networks, interoperability refers to the ability to carry out efficient interactions (i.e. exchange information, products and services) between enterprise systems at least three enterprise layers, i.e. data, services and processes, considering the semantics defined in a given context (Doumeingts \& Chen, 2003). Interoperability must consider organizational, economic and social aspects, as well as technological changes.

Two integrated enterprises could also be said to interoperate satisfactorily, since the homogeneity of their processes and systems implies a full (and effortless) capacity to collaborate and exchange information (INTEROP, 2008). The problem arises when the enterprises that need to work together do not want to adopt an integrated way of collaborating and employ heterogeneous systems, different tools and procedures, and also different concepts or languages. Moreover, setting up the collaboration must not imply a loss of their independence or of their capacity to continue to work in an autonomous manner or to collaborate with other organizations (Campos, Martí, Grangel, Mascherpa, \& Chalmeta, 2008).

Another important aspect to be taken into account when developing proposals for improvement in interoperability is that, although we usually refer to collaborations with external institutions, it is included (and are even based on) collaborations between different departments or systems that exists within the enterprise itself and which were set up to carry out internal processes. This is defined as intra-interoperability.

In order to establish and define projects for improving interoperability, first it is necessary to evaluate and diagnose the situation in which the enterprise currently finds 
itself and to suggest improvements that support evolution in this field. To measure this situation it is necessary to consider a maturity model.

The term Maturity Model was made popular by the SEI (Software Engineering Institute) when the Capability Maturity $\mathrm{Model}^{\odot}\left(\mathrm{CMM}^{\odot}\right)$ was put forward in 1986 (Dymond, 1995). This maturity model has gradually evolved with each new version and is widely accepted as a guide for evaluating the business processes of an organization (Ahern et al., 2004). Based on this initiative, several maturity models have also been put forward in interoperability research (Ford et al., 2007), (Guedria, Naudet, \& Chen, 2008).

In the last decade different maturity models applied to interoperability have been proposed, most of them in the technological field and also taking into account all the different layers of the enterprise (Kasunic \& Anderson, 2004).

In most cases the existing maturity models only define the interoperability enterprise levels, there are some maturity models that include a set of good practices advising enterprises about how to become interoperable (Daclin, Chen, \& B. Vallespir, 2006a), (Pardo \& Burke, 2008). However, in general, the proposals do not go into great depth regarding which aspects need to be evaluated, how to measure them in order to assign a level of maturity, and how to improve this level, if it is considered necessary to do so. Next sections provide a more detailed description of the interoperability maturity models that, because of their relevance and contents, were taken as the starting point for the proposal in this work.

\subsection{Levels of Information Systems Interoperability (LISI)}

The first significant initiative carried out to measure interoperability was proposed by the DoD C4ISR Working Group and entitled Levels of Information Systems 
Interoperability (LISI) (C4ISR, 1988). The aim of LISI was to establish (and, consequently, to improve) the maturity of the information systems used by the US Department of Defense in joint actions implemented between different military units. LISI provides a maturity model including the levels definition, the necessary processes to identify the interoperability needs and how to enable the information systems to support those needs.

LISI proposes five levels of maturity: Isolated, Connected, Distributed, Domain and Enterprise. To establish these levels of maturity, it defines four areas of interest named PAID, which stands for Procedures, Applications, Infrastructure and Data. This maturity model establishes a first approach in order to develop a full maturity model, although the proposal is essentially focused on the technological platforms that support information systems and do not cover all the areas of interest that must be taken into account in enterprise interoperability, such as knowledge or semantic.

\subsection{Organizational Interoperability Maturity Model for C2}

The model proposed in (Clark \& Jones, 1999) completed the LISI model by extending it into organizational layers. It was proposed in the Command and Control (C2) framework of the Allied Joint Task Forces. It proposed five layers of support and a maturity model that established the following levels (from best to worst): unified, combined, collaborative, ad hoc, and independent.

These levels were aligned with the LISI levels, and attributes for organizational interoperability were defined for each one. However, no method of measuring the level achieved is described in detail. 


\title{
2.3 Enterprise Interoperability Maturity Model ATHENA (EIMM)
}

\author{
ATHENA, Advanced Technologies for Interoperability of Heterogeneous Enterprise \\ Networks and their Applications, (ATHENA, 2006a), is a project of the European \\ Union that proposes an Enterprise Interoperability Maturity Model called EIMM
} (ATHENA, 2006b).

EIMM defines five enterprise interoperability levels, i.e., Performed, Modeled, Integrated, Interoperable and Optimizing. EIMM helps to assess an organization's level of maturity concerning the use of enterprise models as well as, the capability of these models to enable the company to establish collaborations. Based on an EIMM assessment, companies will be guided to choose the right concepts in order to improve their capabilities, by taking into account the environment and enterprise challenges (Berre et al., 2007). Although EIMM state that parameters and methods must be defined to measure interoperability, no complete proposal has been put forward showing the steps to be followed or the methods and tools to be used to carry out this measurement.

\subsection{Barriers Driven Methodology Maturity Model}

Another maturity model is proposed as a result of the Barriers Driven Methodology (Daclin, Chen, \& B. Vallespir, 2006b), (Guedria et al., 2008). This methodology considers three types of measurement:

- The interoperability potential measurement, which is concerned with the ability of an enterprise to interoperate without the need to know its interoperation partner and, consequently, with identifying a set of characteristics that have an impact on interoperability. The aim is to measure the intrinsic capabilities of an enterprise to interoperate with an unknown partner. This 
measure must evaluate the accessibility and facilities an enterprise has to set up collaborations with others, the use of standards, the organization's flexibility in the use of enterprise modeling, etc.

- The interoperability compatibility measurement evaluates a current relationship between known stakeholders. In other words, it is measured while the interoperability project is being carried out in order to establish how well two partners are suited to be able to interoperate.

- The interoperability performance measurement has to be set up during the operational phase to evaluate aspects related with the costs involved in implementing interoperability between two enterprises or systems in terms of time or economic investments.

The levels defined to support interoperability potentiality measurement are: Isolated, Initial, Executable, Connectable and Interoperable. Table 1 shows the description for each of these levels.

Interoperability potential (Chen et al., 2008) measurement (Guedria et al., 2008) of an enterprise will evaluate how prepared it is to establish, smoothly and efficiently, collaborations with possible partners, relations with current and new customers, business agreements with suppliers, and communication with governmental or financial institutions. Being prepared and having a high level of interoperability potentiality is a critical factor that will enable the enterprise to adapt to changes and new needs or requirements from the market in a dynamic manner, which will result in better business outcomes.

Although Barriers Driven Methodology highlight the importance of evaluating the interoperability potentiality measurement of enterprises as a critical aspect for carrying 
out improvement projects, it does not put forward or define a proposal as regards how to measure this interoperability potentiality in a practical way.

\subsection{The Levels of the Conceptual Interoperability Model}

In this proposal (Tolk \& Muguira, 2003), the levels of interoperability are defined considering the data to be interchanged and the interface documentation. These levels are:

- Level 0: System Specific Data

- Level 1: Documented Data

- Level 2: Aligned Static Data

- Level 3: Aligned Dynamic Data

- Level 4: Harmonized Data

This model tries to build a bridge between conceptual design and technical design for interoperability. However, although the proposal provides a different view of interoperability, these maturity levels are defined regarding only the interoperability of data and the conceptual design of the databases.

\section{PROPOSAL OF A MATURITY MODEL FOR INTEROPERABILITY POTENTIAL MEASUREMENT}

In order to improve and increase interoperability potential, that is, the level of preparation of an enterprise to establish an efficient collaboration with possible and unknown enterprises, it is necessary to measure the current situation in order to identify

URL: http:/mc.manuscriptcentral.com/uism Email: janice.sipior@villanova.edu 
problems and opportunities for improvement, as well as to define an action plan. To do this, being able to use a maturity model would be a valuable aid.

A review of the literature on maturity models and interoperability showed that there are different maturity models that propose levels that can be assigned to an enterprise regarding its interoperability maturity. Yet they do not provide parameters with which to measure interoperability or interoperability potential (taking into account different views such as processes, departments, technology, etc.) neither a methodology to address the process of measurement and to help identify the improvements needed to increase the level of interoperability. Consequently, these are important problems concerning the measurement of the interoperability potential that remain unsolved. To help to solve this problem, a maturity model called MM-IRIS, which would serve as a support in the evaluation process for detecting the current interoperability potential of an enterprise, was developed and applied to a practical case.

This model includes:

- A set of parameters that take into account different enterprise views.

- A methodology that offers guidance on how to evaluate which level of maturity of interoperability potential is achieved for each of the processes the enterprise performs.

This proposal completes and improves the current status of research in this field, where the methods and maturity models analyzed only define levels and attributes, but do not describe how to measure or evaluate these levels.

The research method that was used to obtain the MM-IRIS maturity model was the qualitative case study method. The work plan that was followed was based on the inductive analysis of qualitative data (Yin, 1994), adapted to proposals for the generalization of a theory from an interpretative investigation (Walsham, 1995), and 
consisted in the following seven stages: Definition of research purposes; Literature review; Case selection; Definition of research methods and resources; Fieldwork; Formulation of the MM-IRIS maturity model; Verification of the rigor and quality of the study.

The enterprise views, the parameters and the methodology defined are described in the following subsections.

\subsection{Enterprise views}

The level of maturity in interoperability potential is not homogeneous in all the enterprise and it is therefore necessary to define different views that make it possible to detect the different levels attained in each case. In the MM-IRIS the following views are proposed: Business, Process Management, Knowledge, Human Resources, ICT and Semantics. These views are defined taking into account the literature dealing with enterprise interoperability and especially with the measurement of interoperability. The authors' own experience in real enterprise interoperability projects is another valuable asset that is also considered. Thus, the business, process management, and ICT views were adopted from (Chen \& Daclin, 2007); the semantic and knowledge views were included following a review of the paper on enterprise layers by (Doumeingts, 2003), who stressed the importance of these aspects in enterprise interoperability; and finally, the human resources view was defined based on the lessons learned in enterprise interoperability projects carried out by the authors, since the previous views did not take into account people's capacity for interoperability, which is an essential aspect for achieving full interoperability.

- The Business (BS) view considers the strategic aspects related with the interoperability: culture, mission, vision, values, and the economic, social and 
environmental policies of organizations. In order to interoperate, enterprises must have aspects that favor collaborations defined within their strategy.

- The Business Process Management (BPM) view includes the work methods (and therefore aspects related to productivity and costs). Interoperability can only be reached when it is based on an efficient interaction with the processes of other enterprises.

- The Human Resources (HR) view, which considers the skills, competencies, roles, culture, collaborative capacity, and so forth, of employees who participate in interoperability processes.

- The Knowledge (K) view, which includes establishing a knowledge management system with which to identify, extract, represent, process and exploit the knowledge that facilitates efficient cooperation among the different enterprises.

- The Information and Communication Technologies (ICT) view, which helps applications, data and communication components to interconnect automatically. The Semantics (S) view is used to facilitate the understanding of the terminology used by the enterprises that wish to collaborate, that is to say, it considers the aspects needed to ensure that the information is interpreted in the same way.

The parameters defined in the ICT view will concern, above all, general technological issues in the enterprise. In each of the other views, use of the ICT as a support will be valued. 


\subsection{Measurement parameters of each view}

Interoperability is accomplished if it is achieved in all areas or views of the enterprise. Hence, carrying out a separate evaluation of each of them will make it possible to detect where there is a greater need for improvement and to define projects that are suitable for each case.

In defining the measurement parameters for each of the views, the issues and concepts identified in the literature to support and improve interoperability have been considered:

- Interoperability domains to provide enterprise interoperability solutions defined by (IDEAS, 2006) and (INTEROP, 2008): Enterprise Modeling (EM), which deals with the representation of the inter-networked organization and considers how to ensure interoperability between different models; Architecture and Platforms (A\&P), which considers the necessary technology to implement interoperable applications; and ontologies (ONTO), which ensure that the semantics used are understandable by the two systems.

- Enterprise layers where interoperability must be achieved: business, knowledge, ICT, and semantics (Doumeingts \& Chen, 2003).

- Barriers to be overcome (Chen \& Daclin, 2007): technological, conceptual and organizational.

- Interoperability focused on different industrial sectors, such as e-government (Alvarez Sabucedo \& Aido Rifon, 2010).

- Performance measurement applied to business processes (Alfaro, RodriguezRodriguez, Verdecho, \& Ortiz, 2009) and interoperability (Blanc, Ducq, \& Vallespir, 2007).

These works highlight the importance of measuring interoperability, the aspects to be considered in measuring it and the criteria for setting the levels of interoperability. 
These results, together with the findings from previous work carried out by the authors in the fields of methodologies and interoperability (Campos et al., 2008), of knowledge modeling (Grangel, Chalmeta, \& Campos, 2007), and performance measurement systems (Chalmeta \& Grangel, 2005), were then used to define the indicators for measuring interoperability potential.

Next, the parameters for each view are defined and justified. For each set of parameters the relationships between each interoperability potential level and the measurement results of the parameters are shown in Tables 2, 3, 4, 5, 6, and 7.

\section{Parameters for the business view}

The first step to measure the enterprise interoperability potential maturity is to establish the enterprise's vision and strategy when faced with the challenges of reaching a suitable level of interoperability. In order to define the parameters of this view, it is deemed necessary to measure those aspects related with the strategic plans of the enterprise that have repercussions on its capacity to establish collaborations. Having certifications endorsed by official, especially international organizations, such as the International Standards for Business, Government and Society (ISO), (http//www.iso. org/iso/iso_catalogue.htm), will allow collaborations to be established more clearly and quickly. Processes in enterprises will be organized and will comply with standards that other firms can rate positively when it comes to establishing new businesses or maintaining existing ones. Next parameters are detailed and Table 2 shows each interoperability potential level and the measurement results.

- Sustainability and quality policies, certified by official bodies: an enterprise that already has, or is implementing, these standards will be able to establish collaborations more efficiently. 
- Any certifications in the area of quality and sustainability that the enterprise already has will be evaluated, as will those that are pending but are currently being assessed. A study will be conducted to compare the minimum standards that the enterprise must have by law and those that exist at state, autonomic and international level.

- The capacity/willingness to adapt to organizational, technological and social changes. Flexibility and adaptation to change can be evaluated by the number of training schemes that exist in the enterprise related to the incorporation of new business models or new technologies. Projects that have been carried out, or are due to be carried out soon, that take into account social aspects such as sustainability or gender equality will be valued. The new business approach toward aspects with social repercussions is an added value that can be decisive when it comes to establishing relations with public bodies that value such issues.

- Strategy as regards the use of technologies as a support to aid in collaborations with other enterprises. Projects carried out in recent years on improvements in the technological field that may result in better interactions with other firms and the proposals included in the strategic plans will be evaluated.

- Policies with respect to the use of technological and information standards. Whether or not this type of policy exists and the willingness to adopt them will be key factors that must be evaluated in order to know the extent to which the enterprise is ready to interoperate.

- Policies of (social, technological, etc.) evaluation of possible partners prior to establishing relations. The existence of procedures that allow the capacities and characteristics of a possible collaborator to be ascertained and evaluated, taking into account not only technological aspects but also organizational and social 
responsibility, will make it possible to know whether the company is prepared to be more efficient when selecting future collaborations.

- Contractual policies regarding collaborations with other bodies. Evaluating the existence of conditions beyond those contemplated by the law will make it possible to know whether the enterprise controls and establishes its own criteria when it comes to interoperating with another enterprise.

\section{Parameters for the Process Management view}

The processes view will evaluate the level of formalization that exists, the documents and use of enterprise modeling languages, the capacity to exchange and make public the parts of the processes in which collaborations with other firms may take place. The parameters considered are:

- Identification of processes in which there is some collaboration with external institutions: it is necessary to find out whether the processes are identified, whether they are documented and within the collaborators reach, and whether they are represented in some enterprise modeling standard.

- Formalization of processes: tacit, documented, modeled procedures. Evaluations will be conducted to determine whether procedures have been defined or whether activities are simply carried out as they are learnt from experience. If the processes have been formally defined, they will be evaluated to determine whether they are documented, together with the modeling languages and tools used to represent them.

- Process planning: studies will be conducted to examine whether processes are planned and, if so, the deadlines that are set and the level of detail to which they are planned. 
- Quality measurement and control: in processes that involve collaboration with external institutions, in which cases methods and indicators have been developed to measure the quality and to control processes.

Table 3 shows each level of interoperability potential and the measurement results for these parameters.

\section{Parameters for the Human Resources view}

One aspect that is fundamental in order to have a good level of interoperability potential is how prepared the workers are, the plans for training that exist in the company, and how easily human resources adapt to changes. The parameters to be measured in this view are:

- Organizational structure: there is a well-defined and documented structure with a clear hierarchy and allocated functions.

- Assigned roles, flexibility to exchange jobs.

- Training the enterprise has a training plan that takes into account different levels and contents depending on the tasks and human resources.

- Evaluation and control: assigning resources to follow up, supervise and make processes known.

Table 4 shows each level of interoperability potential and the measurement results for these parameters.

\section{Parameters for the Knowledge view}

The knowledge view covers aspects concerned with how knowledge is managed and transmitted, not only internally, but also how it is identified and transmitted to possible collaborators that need to use it. The interoperability potential of an enterprise will, in turn, be marked by the level of maturity that exists in the management of its knowledge, 
since greater specification of enterprise knowledge makes the company better prepared to establish efficient collaborations with possible stakeholders that seek to interoperate with it.

Furthermore, companies have a greater level of maturity in this field if they have the capacity to apply the ICT to enterprise knowledge management by a Knowledge Management System (KMS). The first aim would be to identify the knowledge that is going to be managed by the system, that is to say, the target knowledge.

The parameters are:

- Knowledge that is exchanged and points of interaction, which will make it possible to detect the existence of at least tacit knowledge in the processes of the enterprise.

- Channels of knowledge to evaluate the use of suitable methods and technologies for the exchange of information.

- Existence of a Knowledge Management System.

- Identification of the conceptual blocks of knowledge and the target knowledge that the enterprise wishes to manage; thus, evaluations are carried out to determine whether possible stakeholders and points for exchanging or sharing knowledge have been identified.

- Type of conceptual blocks on which the target knowledge has been defined.

- Identification of the explicit and tacit sources of knowledge.

- Knowledge modeling that has been identified, so that the enterprise's target knowledge concerning its processes, products, resources, suppliers, customers, etc. is made explicit.

- Exploitation of knowledge by means of a KMS. 
Table 5 shows each level of interoperability potential and the measurement results for these parameters.

\section{Parameters for the ICT view}

Information technologies play a fundamental role in achieving a high level of maturity. The use of standard platforms and technologies is essential to be able to interoperate and to be able to open up new collaborations efficiently; companies not prepared to cooperate with each others using ICT and inter-enterprise applications will fall through their business (Hoving, 2007). The parameters are:

- Technological resources for communication: which ones exist, which ones are standard.

- Executive, decisional and computer systems for the integrated management of information: whether they exist, are used and training is carried out in this issue.

- Planning of technology needs in order to support collaborations.

- Policies for the development of platform-independent technologies that can be adapted to different systems.

- Infrastructures to support process management (Workflow, EM tools, etc.), control of both internal processes (auditing, register of the use of the services) and those of external entities.

- Technology utilities for public use if they exist, their use is recorded or they are planned for the future.

Table 6 shows each level of interoperability potential and the measurement results for these parameters. 


\section{Parameters for the Semantics view}

The Semantics view is concerned with ensuring that the precise meaning of any exchanged information can be understood by any other processes, people, collaborative external enterprises and ICT applications. So, taking in account that Ontology domain addresses the semantics necessary to ensure interoperability (Duque, Campos, JimenezRuiz, \& Chalmeta, 2009), the high level of maturity will be achieved if the use of ontologies is applied to support collaborations and internal business management. The proposed parameters are:

- Databases and contexts where these databases operate. Existing mappings.

- Collections of terms: whether they exist and whether there are methods to collect and process them.

- Planning technologies and infrastructures for supporting these collections of terms and mappings.

- Mapping between own collection of terms and public thesaurus.

- Planning about acquiring (ontology tools and training human resources on the use of them.

- Ontology developed to support both semantic webs and collaboration processes.

Table 7 shows each level of interoperability potential and the measurement results for these parameters.

\subsection{Maturity measuring methodology}

In order to evaluate the interoperability potential level, a step based methodology has been developed. 
The phases of the methodology will be carried out progressively and iterations can also take place. This means that it is necessary to repeat or improve part of the results from the previous phases as the measurement project advances.

During of the application of the methodology it is necessary to collect information. The main technique employed is a questionnaire that must be developed considering the parameters already defined for each view. Each parameter is transformed into one or several questions on a questionnaire, which is adapted to the enterprise taking into account its business processes and the (current and potential) collaborations that were previously identified and which may be internal (between departments) or with external entities. The questions can be quantifiable in absolute values, scored on a given scale (from 1 to 5), with yes/no answers or as a percentage (for example $\%$ of employees with a higher education). In any case, each question must include a section where the respondent can describe whether this aspect needs improving or not, or if the current situation is seen to be sufficient.

While the questionnaire is being developed, weightings must be defined for each of the questions and must be established which results correspond to each of the levels, taking into account the previous descriptions produced in the tables for each of the views.

Next, each phase of the methodology is described in greater detail. Figure 1 shows graphically the phases proposed and the main results obtained from each phase, according to the enterprise views defined.

\section{Phase 1: Project planning}

The basic aim of this phase is to define the conceptual aspects of the enterprise as regards interoperability, taking into account the business view and the strategic and cultural goals. 
Since it represents the beginning of the project, it is necessary to define the human resources that will be involved in the work, to establish the scope (areas and/or processes) to be evaluated and possible restrictions concerning time or costs, and to estimate and schedule the project in order to control it.

In this phase the parameters of the business view are taken into account in order to develop questionnaires and interviews to evaluate the strategic plans, the policy of the enterprise and the preparation degree to cope with the challenge of improving its interoperability potential.

The techniques to be utilized in this phase are information collection techniques, above all face-to-face interviews with company managers and more especially with those who are more deeply involved in the strategic and improvement projects at the enterprise, such as quality, information technologies or R\&D managers. Documents about quality standards, the company mission and its vision are also collected. This first result makes it possible to evaluate whether it is feasible to continue with the rest of the phases or if it would be better to limit the study and measurement to the interrelations among the departments in order to carry out a preliminary assessment of internal interoperability in the company. In an enterprise where the business strategy does not include fundamental aspects such as policies on external collaboration or strategies for improving these collaborations or the ICT, there will be no point in evaluating their interoperability potential maturity in further detail in the remaining views.

The results are: planning, where criteria and priorities are established in order to delimit the scope of the project and its feasibility; definition of the strategy for carrying out the following phases of the measurement project, and evaluation of interoperability potential in the Business view. 


\section{Phase 2: Definition and classification of collaborations}

After identifying the fact that the enterprise has established or planned certain policies or needs in relation to interoperability as part of its strategic plans, the second phase has three basic aims:

1. To identify and classify the internal collaborations for each of the processes.

2. To identify and classify the external entities with which situations of interoperability take or may take place, considering each of the enterprise's business processes.

3. To identify the current collaborations with these external entities, taking into account processes and departments involved.

The first step is to study and review the organizational structure of the company, the process map and to identify the collaborations that exist between each department for each of the enterprise's business processes.

The process map and the organizational structure are then used to identify and classify the types of collaboration that can be set up and the stakeholders, for example financial institutions, governmental entities, large, small or medium-sized supplier enterprises and large, small or medium-sized customers.

It is necessary to have reliable up-to-date information about the business processes that the company carries out, the departments involved and its business strategy with respect to other supplier or purchaser entities which it may interoperate with.

Information about the organization and the hierarchical structure of the company and its process map will be used as working documents. The results of identifying and classifying the departments involved in each process are:

- An Internal collaboration matrix that shows, for each process, what department is responsible and what interactions it establishes with other departments. 
- An External collaboration matrix that considers what the current interactions with external entities are for each process and department.

- A list of Current partners or collaborators and potential ones.

\section{Phase 3: Measurement and collection of results}

The main result from this phase is a questionnaire for evaluating interoperability potential maturity, adapted to the structure and processes of the enterprise. The questionnaire is organized taking into account the collaboration matrices that were obtained earlier and the parameters for the Process Management, HR, Knowledge, ICT and Semantics views. Several questions are defined for each of the parameters. The criterion for defining the number of questions and their content is that they should allow the parameter to be evaluated within the context of the interoperability of the enterprise. For example, in most cases it will be necessary to define questions that make it possible to measure whether the parameter exists, whether it is known, whether it has been documented, whether it is modeled, or whether it is supported by the technological tools or standards. Different iterations may occur until a final questionnaire is obtained, as described in (Palomares, Campos, \& Palomero, 2010). Two critical aspects in constructing the questionnaire are that it must be both easy to understand and simple to answer. In order to make it easier to understand, it is essential to get top and middle management involved so that the questions can be adapted to the terminology and peculiarities of the enterprise. To make it easier to answer, the questions were designed in such a way that they can be answered with a set of values ranging from 1 , for the worst situation, to 5, for the best one. There is also a column for the interviewee to add whether the situation needs improving or if it is considered to be satisfactory.

The outcomes in this phase include:

- Review of the documents obtained in Phase 1. 
- Definition of tactical goals.

- Questionnaires to evaluate the interoperability potential for each process and collaboration.

- Completed questionnaires.

\section{Phase 4: Analysis and quantification}

With the answers given in each of the questionnaires, and taking into account the levels and questions assigned to each parameter, a detailed analysis and quantification of results must now be performed to assign a level of interoperability potential. Each question is answered with a value and a comment on the need for improvement, and then the quantitative values need to be evaluated and analyzed.

Since the study is conducted by processes and views, one particular process can have a high level of interoperability in one view and with respect to one group of collaborators, but at the same time have shortcomings or a low level in another aspect or view.

In this phase data analysis techniques and cost/benefit analysis must be used, and the members of the work team must meet to compare results with the users. The results are the interoperability potential levels and the identification of needs of improvement, both of them detailed for each process, collaboration (internal, current and potential with external entities) and view.

\section{Phase 5: Proposals for improvement}

Once the results from the questionnaires have been quantified, an analysis must be conducted to study and evaluate the points or processes where the level of interoperability reached needs to be improved. In this analysis the strategic aspects identified initially in the first activity must be taken into account. The result of this 
evaluation is a proposal for projects arranged in order of priority, in which the current "AS-IS" situation and the one that is sought (or "TO-BE") are both established.

Traditional cost/benefits analysis is a very useful tool to define the priority of the projects within the interoperability project. However, organizational, technical and operational aspects should also be considered.

The results are a proposal including: medium and long-term interoperability improvement projects, interoperability future needs, and a viability analysis.

\section{A CASE EXAMPLE}

The MM-IRIS maturity model was applied to a large textile enterprise to be validated and provides some practical aspects that can guide similar applications in other cases. The enterprise is a large company from the textile sector that is firmly rooted in both the Spanish national and the international markets. It has a complete supply chain, logistics centers separated from its centers of production, and suppliers of finished and semifinished products in different countries.

It has different types of customers including micro/small, medium-sized and large enterprises, with a wide range of technologies and policies. Like any company, it has relations with public or governmental and financial institutions.

An evaluation of the firm's potentiality to interoperate both with customers and with suppliers of raw materials and intermediate or finished products will allow have a number of benefits for the company. For example, it will be able to develop strategic plans that help it to improve these collaborations, make more reliable choices (in terms of results) regarding possible partners in interoperability projects, and evaluate certain aspects that, due to the production and supply structure, are crucial to the enterprise. 
Next the procedure adopted for the application of the MM-IRIS in this company is described.

\section{Project planning (Phase 1)}

Initially an introductory meeting was held with managers from the company and those in charge of a number of different departments, which in this case mainly involved the Information System and Quality departments. At this meeting the basic concepts and aims of the work to be carried out were explained and the benefits that the enterprise would gain from collaborating in the project were also outlined. More specifically these benefits were: (1) a reappraisal of its organizational structure and process management; (2) measurement of the level of interoperability potential; (3) diagnosis of its situation in this area; and (4) definition and study of the feasibility of projects for improving interoperability in the short and medium term.

Following this meeting a work team was set up that included both research personnel and management staff from the company's Quality and Information Systems departments.

In order to define the conceptual aspects and to establish the scope of the project, a preliminary research task was conducted. This task included a revision of the enterprise process map and an evaluation of what the strategic view about interoperability was.

To be able to evaluate these strategic aspects, the business view parameters were considered and a first questionnaire for high level managers was developed. This questionnaire had two main objectives.

- To identify strategic needs, capacity and willingness with regard to interoperability aspects. 
- To determine the current organizational structure, departments, responsibilities and policies about interoperability.

A subset of the questions which were applied is shown in Table 8. In this case the possibility of including comments in the questions was also considered, so that the managers could offer more detailed information.

Explicit results were the project plan, including the scope, and the main departments and staff involved. Other outcomes were:

- Recognition that the enterprise applies interoperability principles (although not explicitly) to carry out different transactions and processes involving external collaborators, such as financial and governmental institutions, suppliers and customers.

- Identification that a suitable but insufficient policy regarding the use of ICT as a support for business processes was also found to exist.

- Revision of the documentation and the information about the organization of the company.

\section{Definition and classification of collaborations (Phase 2)}

During the first phase, the project team reviewed the current process map of the enterprise. This yielded the following results:

- An update of the process map, after the revision.

- Identification of the processes that produced collaborations both between different departments as well as with external entities.

- A first definition of the most significant groups of potential collaborators (see Table 9). In order to produce a classification of current and potential partners it 
was taken into account economical aspects, governmental institutions, law impositions and commercial agreements.

The information was then used to create the internal collaboration matrix and the external collaboration matrix. Figure 2 represents part of an example of these two matrices; in the rows it shows the enterprise processes considered within the scope of the project and the department responsible for each process. The letters represent each department for the internal collaboration matrix, and the classification of current and potential external collaborators, for the external collaboration matrix. The managers involved in the project filled in both matrices, and the result was the identification of collaborations, between departments in the first one, and with external entities in the second.

\section{Measurement and collection of results (Phase 3)}

In order to produce the questionnaires, some specific characteristics of the enterprise were considered. In particular, this company is organized in departments that are responsible for processes. The enterprise business processes, which were identified in the previous phase, provide information about what kind of collaborations are carried out between departments as well as with external entities. Therefore, in this case, it was decided that a separate questionnaire should be created for each department to ask about the processes under its responsibility.

For each department one specific collaboration matrix was defined that took into account only those processes under its responsibility and its own collaborations, including both internal and external ones. Figure 3 shows an example for the Purchasing department. 
Therefore, the second step was to define questions for the parameters of each view. Each question is answered on the specific department's collaboration matrix. The current situation can be valued from 1 (the worse situation) to 5 (the best). The users can also identify whether improvements are needed in the column "OK/NO". As an example, Table 10 shows the questions developed for the ICT view, and Figure 4 shows the final structure of the questionnaire.

After several iterations to obtain an accurate and customized questionnaire, the final version was applied with the collaboration of the managers in the Quality and Information Systems departments. This fact generated more confidence in the managers of other departments who were interviewed and enabled more reliable results to be obtained more efficiently.

\section{Analysis and quantification (Phase 4)}

After obtaining the filled-in questionnaires, the results were analyzed and quantified. One implicit result was the positive evaluation of the efficiency and correctness of the questions that were posed.

Statistics and comparisons of data were carried out by parameters where the answers could be grouped and quantified by processes, views and departments. A qualitative report was also drawn up that included not only an evaluation of the answers, but also a list of the points in which the greatest need for improvement was detected.

The final result was a detailed evaluation of the interoperability potential for each process and view. As an example of the results, Figure 5 shows a bar graph that includes, for some of the enterprise departments and two processes, the results obtained in three of the views. 


\section{Proposals for improvement (Phase 5)}

In addition to this diagnosis in relation to interoperability, a proposal was also put forward for short- and long-term improvements in those departments where the most urgent needs were detected, including the corresponding feasibility study for the shortterm projects that were identified.

\section{Lessons learned}

The MM-IRIS maturity model provides a series of clear steps to be followed and a set of results that allow a detailed evaluation of the interoperability potential to be performed. At the same time, an analysis of different organizational aspects of the enterprise can also be conducted.

The development of the questionnaire as the central and fundamental task in the evaluation of maturity in interoperability potential must be a job that is carried out in close collaboration with qualified staff from the firm.

The participants in the project noticed that the application of the questionnaire - with open questions - provides subjective results that must be analyzed within the context of the persons that participate. It has also been identified that for the same real scenario different answers can be collected that consider the personal perception of the people interviewed. In this sense it is important to identify the main participants and those who have the knowledge and the capabilities to make decisions. A quantitative method of collecting results would have provided greater accuracy and trust than the qualitative method used. Nevertheless, owing to the characteristics of the parameters and of the non-technological views, the quantitative method cannot be used. It is necessary to take into account aspects that are deduced from meetings and from open questions, where 
managers can suggest improvement needs and bear in mind other specific situations.

However, results will become more reliable as the level of commitment of management goes up and interviewees are informed correctly about the benefits of the project.

In addition to an evaluation of its level of maturity in the area of interoperability potential and the aspects in need of improvement, the enterprise also gained other benefits from applying MM-IRIS, such as:

- Department managers improved their knowledge and training in relation to interoperability aspects.

- Business processes were reviewed, above all those in which it collaborates with external institutions.

- The enterprise was already carrying out some of its collaborations efficiently, but evaluating them by means of this work enabled it to detect aspects that could be improved and that were not being implemented properly.

- Definition and classification of current and potential collaborators.

- Having criteria that enabled it to evaluate and select possible collaborators.

The application of MM-IRIS provides several different benefits, such as:

- Identification of current collaborations and potential stakeholders that may best fit future needs.

- Review of current interoperability situation in all the enterprise views.

- Identification of strategic needs to improve interoperability.

- Design of an interoperability guidance plan where the different projects for improving it are defined.

- From the implementation of the interoperability improvement projects new business opportunities are generated, costs are reduced, and the effectiveness and efficiency of the enterprise are enhanced.

URL: http:/mc.manuscriptcentral.com/uism Email: janice.sipior@villanova.edu 


\section{CONCLUSIONS AND FUTURE WORK}

In order to improve all the aspects that affect the capacity to interoperate, first it is necessary to be able to evaluate the AS-IS situation, considering aspects related with process management, organizational features, human resources and the semantics of the company.

In addition to knowing their capacity to interoperate with known collaborators, enterprises also need to know how prepared they are to establish relations in the future. Evaluating the level of interoperability potential (taking into account all the views of the enterprise with details of each process) enables the company to become aware of its strong points in this field and of the improvement projects it could consider in order to raise better chances of collaboration. To support these objectives, it is necessary to have a set of methodological guidelines to indicate the procedures to be carried out.

The level of interoperability potentiality cannot be improved in such a way that it goes straight from an initial level (level 1) to an interoperable level (level 5); instead it is necessary to implement projects that allow the processes of the enterprise to gradually evolve from one level to the next one. It is also important to note that not all the processes in an enterprise require the same level of interoperability. In some processes a medium level may be sufficient while in others requirements imposed by the market or by other stakeholders may call for the highest level.

Parameters related with the domains of interoperability and with the conditions that the company processes must fulfill in order to be prepared for new collaborations, while also taking into account different views, can be used by the management of an enterprise as a solid foundation on which to carry out diagnoses and proposals for improvement projects. 
With regard to future work in this area, some reflections need to be made on the new proposals that research on interoperability is evolving toward, and more particularly on the recommendations suggested by Future Internet Enterprise Systems Cluster (Community Research and Development Information Service - CORDIS). Research recommendations point to the idea that a new notion of enterprise and enterprise network is arising. Various lines of research that take the new scenario into account have been suggested, e.g. incorporating the role of enterprise culture in enterprise collaboration or the notion of sustainability, including economic, environmental and social dimensions.

Bearing this proposal in mind, the evolution of the work presented in this paper should consider new characteristics for measuring interoperability potential that take into account the aspects of this new approach by including parameters in each view in order to evaluate them.

\section{ACKNOWLEDGEMENTS}

\section{REFERENCES}

Ahern, M., Clouse, A., \& Turner, R. (2004). CMMI Distilled. A Practical Introduction to Integrated Process Improvement. SEI. Addison Wesley.

Alfaro, J. J., Rodriguez-Rodriguez, R., Verdecho, M. J., \& Ortiz, A. (2009). Business process interoperability and collaborative performance measurement. International Journal of Computer Integrated Manufacturing, 22(9), 877-889. doi:10.1080/09511920902866112.

Alvarez Sabucedo, L., \& Aido Rifon, L. (2010). Managing Citizen Profiles in the Domain of e-Government: The cPortfolio Project. Information Systems 
Management, 27(4), 309-319. doi:10.1080/10580530.2010.514181.

ATHENA. (2006). Advanced Technologies for Interoperability of Heterogeneous Enterprise Networks and their Applications IP (IST-2001-507849). Retrieved from http://interop-vlab.eu/ei_public_deliverables/athena-deliverables.

ATHENA. (s.d.). Interoperability Framework and Services for Networked Enterprises. Retrieved from http://interop-vlab.eu/ei_public_deliverables/athenadeliverables/A4/d-a4.2/.

Berre, A., Elvesaeter, B., Figay, N., Guglielmina, C., Johnsen, S., Karlsen, D., \& et.al. (2007). The ATHENA Interoperability Framework. In Enterprise Interoperability II. Springer.

Blanc, S., Ducq, Y., \& Vallespir, B. (2007). Evolution management towards interoperable supply chains using performance measurement. Computers in Industry, Volume 58, Issue 7, 720-732.

C4ISR. (1988). C4ISR Architecture Working Group. Levels of Information Systems Interoperability (LISI).

Campos, C., Martí, I., Grangel, R., Mascherpa, A., \& Chalmeta, R. (2008). A Methodological Proposal for the Development of an Interoperability Framework. In Model Driven Interoperability for Sustainable Information Systems (MDISIS'08) (CAiSE'08), CEUR-WS (Vol. 340, pp. 47-57).

CORDIS. (s.d.). Community Research and Development Information Service. Retrieved from http://cordis.europa.eu/fp7/ict/enet/publications_en.html.

Chalmeta, R., \& Grangel, R. (2005). Performance Measurement Systems for Virtual Enterprise Integration. International Journal of Computer Integrated Manufacturing, 18, 73-84.

Chen, D., \& Daclin, N. (2007). Barriers Driven Methodology For Enterprise 
Interoperability. In IFIP International Federation for Information Processing. Establishing The Foundation of Collaborative Networks, IFIP International Federation for Information Processing (Vol. 243, pp. 453-460). Springer Boston.

Chen, D., Vallespir, B., \& Daclin, N. (2008). An Approach for Enterprise Interoperability Measurement. In Model Driven Information Systems Engineering: Enterprise, User and System Models (Vol. 341, pp. 1-12).

Clark, T. \& Jones, R. (1999) Organisational Interoperability Maturity Model for C2 Command And Control Research And Technology Symposium (CCRTS)

Daclin, N., Chen, D., \& Vallespir, B. (2006a). Enterprise Interoperability Measurement - Basic Concepts. In EMOI - INTEROP'06 Enterprise Modelling and Ontologies for Interoperability, CEUR (Vol. 200).

Daclin, N., Chen, D., \& Vallespir, B. (2006b). A methodology to develop interoperability of enterprise applications. Information Control Problems in Manufacturing, 2006, 591-596.

Doumeingts, G., \& Chen, D. (2003). Basic Concepts and Approaches to Develop Interoperability of Enterprise Applications. In L. M. Camarinha-Matos \& H. Afsarmanesh (Eds.), PRO-VE, IFIP Conference Proceedings (Vol. 262, pp. 323330). Kluwer.

Duque, A., Campos, C., Jimenez-Ruiz, E., \& Chalmeta, R. (2009). An Ontological Solution to supprot Interoperability in the Textile Industry. In M. V. S. R. Poler (Ed.), Second IFIP WG 5.8 International Workshop, IWEI 2009 (Vol. 38, pp. 38-51).

Dymond, K. (1995). A guide to the CMM, Understanding the capability maturity model for software. Process Inc US.

Ford, T. C., Colombi, J., Graham, S., \& Jacques, D. (2007). A Survey on

URL: http:/mc.manuscriptcentral.com/uism Email: janice.sipior@villanova.edu 
Interoperability Measurement. In 12th ICCRTS International Command and

Control Research and Technology Symposium. Adapting C2 to the 21st Century.

Grangel, R., Chalmeta, R., \& Campos, C. (2007). A Modelling Framework for Sharing Knowledge. Lecture Notes in Artificial Intelligence. Springer Verlag, 4693, 1230-1237.

Guedria, W., Naudet, Y., \& Chen, D. (2008). Interoperability Maturity Models - Survey and Comparison -. Lecture Notes in Computer Science, 5333, 273 - 282.

Hoving, R. (2007). Information Technology Leadership Challenges - Past, Present, and Future. Information Systems Management, 24(2), 147-153. doi:10.1080/10580530701221049

IDEAS. (2006). Interoperability Development for Enterprise Application and Software Project, (IST-2001-37368). Retrieved from http://cordis.europa.eu/fp5/home. html.

INTEROP. (2008). Interoperability Research for Networked Enterprises Applications and Software NoE (IST-2003-508011). Retrieved from http://interopvlab.eu/ei_public_deliverables/interop-noe-deliverables

Kasunic, M., \& Anderson, W. (2004). Measuring Systems Interoperability: Challenges ans Opportunities. Carnagie Mellon University and Software Institute.

Palomares. N., Campos, C., \& Palomero, S. (2010). How to develop a questionnaire in order to measure interoperability levels in enterprises. In Enterprise Interoperability IV.Making the Internet of the Future for the Future of Enterprises (pp. 387-396). Presented at the IESA 2010, Coventry (UK): Springer Verlag.

Pardo, T. A., \& Burke, G. B. (2008). Improving Government Interoperability: A Capability Framework for Government Managers. The Research Foundation of 
State University of New York.

Tolk, A., Muguira, J.A. (2003). The levels of conceptual interoperability model. In: Fall Simulation Interoperability Workshop, USA (September 2003)

Walsham, G. (1995). Interpretive case studies in IS research: nature and method. European Journal on Information Systems, , 4, 74-81.

Yin, R. (1994). Case Study Research: Design and Methods. Applied Social Research Methods ( $2^{\circ}$ ed.). Newbury Park, CA: SAGE.

URL: http:/mc.manuscriptcentral.com/uism Email: janice.sipior@villanova.edu 
Table 1. Interoperability potentiality levels from (Chen \& Daclin, 2007). 


\begin{tabular}{ll}
\hline Level & Description \\
\hline Isolated & Non-existent and are not included in the short- or long-term goals \\
\hline Initial & Exist tacitly and are considered for inclusion within medium- and \\
& short-term goals \\
\hline Executable & Partially existent and are included in the strategic goals and plans of \\
& the enterprise \\
\hline Connectable & Exist and are planned \\
\hline Interoperable & Exist, are planned and are taken into account within the enterprise's \\
& plans for continuous improvement
\end{tabular}

Table 2. Interoperability Potential levels and results for Business view. 


\begin{tabular}{ll}
\hline Level & Description \\
\hline Isolated & Few amount of tacit or routine knowledge about the organization and \\
& management of processes \\
\hline Initial & Some informal and little-known written specifications exist \\
\hline Executable & Written specifications that are known to those responsible for process \\
& management and a certain amount of short-term planning \\
\hline Connectable & Formal specifications, documents and models exist, and the processes \\
& are planned \\
\hline Interoperable & Standard processes models exist, are improved and are taken into \\
& account in the company's short- and long-term plans for continuous \\
& improvement
\end{tabular}

Table 3. Interoperability Potential levels and results for the Process Management view. 


\begin{tabular}{ll}
\hline Level & Description \\
\hline Isolated & There is no organized structure or plans for training \\
\hline Initial & There is a tacit, informally recognized structure \\
\hline Executable & There is a clear organized structure and the possibility of training of \\
& human resources is taken into consideration \\
\hline Connectable & There is a clear organizational structure and plans for training human \\
& resources \\
\hline Interoperable & There is a clear dynamic organizational structure, plans for \\
& continuous training, and policies and incentives for improvement \\
\hline Table 4. Interoperability Potential levels and results for Human Resources view.
\end{tabular}

Table 4. Interoperability Potential levels and results for Human Resources view. 


\begin{tabular}{ll}
\hline Level & Description \\
\hline Isolated & There is no concern for or definition of knowledge management \\
\hline Initial & The need has been detected and the idea of incorporating the \\
& development of a knowledge management system within the plans of \\
& the enterprise is being considered. There is tacit knowledge \\
\hline Executable & Explicit knowledge exists but it is not managed \\
\hline Connectable & Explicit knowledge and a management system in need of \\
& improvement both exist \\
\hline Interoperable & A knowledge management system and plans for continuous \\
& improvement both exist
\end{tabular}

Table 5. Interoperability Potential levels and results for Knowledge view. 


\begin{tabular}{ll}
\hline Level & Description \\
\hline Isolated & There are no ICT platforms capable of communicating with other \\
& enterprises \\
\hline Initial & There are systems that could connect with others, but large \\
& investments are required to do so \\
\hline Executable & Connections could be made with medium/minimum investments and \\
& the improvements have already been planned \\
\hline Connectable & Connections can be made with the least amount of needs, and there \\
& are already plans for improvement \\
\hline Interoperable & Connections can be made efficiently and with only a few changes. \\
& There are plans for continuous improvement
\end{tabular}

Table 6. Interoperability Potential levels and results for ICT view. 
Table 7. Interoperability Potential levels and results for Semantic view. 
Is the company mission defined, documented and known?

Is the company organizational structure documented and distributed?

Can the business processes easily incorporate changes? (policies, laws, new requirements of the market)

Are the human resources flexible for new roles inside the company?

Is the company certified in ISO standards?

Has the company any other quality certifications? Which ones?

Have any future plans about acquiring other standard certifications been defined?

Is the company infrastructure flexible for the adaptation of new technologies?

Do policies regarding the use of ICT as a support for business processes exist?

Does the company consider implementing any actions to improve the effect on social aspects?

Is the Internet normally used for internal daily communications? Does the strategic plan include improvement of internal communications?

Is it usual to use the Internet for stakeholders' daily communications? Does the strategic plan include improvement of these communications? Are there any policies about the use of these communications? Have any action plans including projects to improve the interaction with stakeholders been defined?

Have any contractual policies to establish collaborations with new partners been defined?

Can new policies be adopted if new collaborations require them?

Have any technological, social and organizational policies been defined to evaluate a new partner for a new collaboration?

Table 8. General questions taking into account Business view parameters. 


\begin{tabular}{ll}
\hline Public entities & Tax- and finance-related government departments \\
& Employment department \\
& International trade administration \\
& Customs offices \\
\hline Financial entities & Banks \\
& External financial entities \\
\hline Service suppliers & Transport agencies \\
& Dealers \\
& Employment agencies \\
\hline Product Suppliers & Final Product suppliers \\
& Raw material suppliers \\
& Packing suppliers \\
\hline Customers & Large companies with special conditions \\
& Small businesses \\
& Medium-sized companies \\
\hline
\end{tabular}

Table 9. Initial classification of current and potential partners. 


\section{Questions}

\begin{abstract}
Planning and policies in IS
1. Have the ICT needs for supporting internal and external interactions with the department been documented?

Computer Systems at the

2. Are there ICT resources for supporting the system operational level activities (mail server, file server, etc.) of the department?

3. Are there computer systems for supporting operational activities of the department?

Computer Systems at the 4. Are there ICT infrastructures for controlling the execution tactical level and further analysis of processes?

Infrastructure to support 5. Are there ICT infrastructures for defining, implementing process management and maintaining the business processes?

6. Are there ICT infrastructures for facilitating external entities' access to public processes?
\end{abstract}


PHASE V

Proposals for improvement

Questionnaire for intra-interoperability an questionnaire for current and potential collaborations (by processes)

\section{PHASE IV}

Analysis and quantification

\section{Enterprise Views}

\begin{tabular}{|c|c|c|c|c|}
\hline Business & $\begin{array}{c}\text { Process } \\
\text { Management }\end{array}$ & $\begin{array}{c}\text { Human } \\
\text { Resources }\end{array}$ & Knowledge & ICT \\
\hline
\end{tabular}

PHASE I

Project

PHASE II

Definition and

Conceptual and strategic aspects

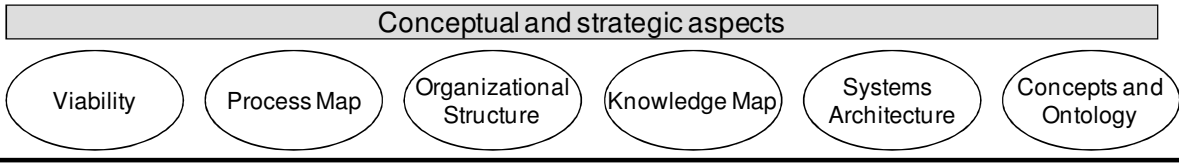

Figure 1. MM-IRIS Methodology and results. 


\begin{tabular}{|c|c|c|c|c|c|c|c|c|c|}
\hline \multirow[t]{2}{*}{ Macro-process } & \multirow[t]{2}{*}{ Micro-process } & \multirow[t]{2}{*}{ Interact with } & \multicolumn{5}{|c|}{$\begin{array}{l}\text { Current } \\
\text { Situation }\end{array}$} & \multirow[t]{2}{*}{$\begin{array}{l}\mathrm{OK} / \\
\mathrm{NO}\end{array}$} & \multirow[t]{2}{*}{ Comments } \\
\hline & & & 1 & 2 & 3 & 4 & 5 & & \\
\hline \multirow[t]{3}{*}{ Product design } & Planning & $\begin{array}{l}\text { Product Design } \\
\text { Dep. }\end{array}$ & & $x$ & & & & $\mathrm{~N}$ & \\
\hline & \multirow{2}{*}{$\begin{array}{l}\text { Supplier } \\
\text { evaluation }\end{array}$} & Suppliers & $x$ & & & & & $\mathrm{~N}$ & \\
\hline & & $\begin{array}{l}\text { Product Design } \\
\text { Dep. }\end{array}$ & & & & $\mathrm{x}$ & & Ok & \\
\hline \multirow[t]{4}{*}{ Purchases } & $\begin{array}{l}\text { Purchase } \\
\text { Order } \\
\text { Preparation }\end{array}$ & $\begin{array}{l}\text { Product Design } \\
\text { Dep. }\end{array}$ & & & $\mathrm{x}$ & & & $N$ & \\
\hline & \multirow{2}{*}{ Receiving } & Quality Dep. & $x$ & & & & & $N$ & \\
\hline & & Suppliers & & & & $x$ & & Ok & \\
\hline & Payment & $\begin{array}{l}\text { Account } \\
\text { Payable Dep. }\end{array}$ & & & & $x$ & & OK & \\
\hline
\end{tabular}

Figure 2. Structure of the internal and external collaboration matrices. 


\begin{tabular}{|c|c|c|c|c|c|c|c|c|c|}
\hline \multirow[t]{2}{*}{ Macro-process } & \multirow[t]{2}{*}{ Micro-process } & \multirow[t]{2}{*}{ Interact with } & \multicolumn{5}{|c|}{$\begin{array}{c}\text { Current } \\
\text { Situation }\end{array}$} & \multirow[t]{2}{*}{$\begin{array}{l}\mathrm{OK} / \\
\mathrm{NO} \\
\end{array}$} & \multirow[t]{2}{*}{ Comments } \\
\hline & & & 1 & 2 & 3 & 4 & 5 & & \\
\hline \multirow[t]{3}{*}{ Product design } & Planning & $\begin{array}{l}\text { Product Design } \\
\text { Dep. }\end{array}$ & & $x$ & & & & $N$ & \\
\hline & \multirow{2}{*}{$\begin{array}{l}\text { Supplier } \\
\text { evaluation }\end{array}$} & Suppliers & $x$ & & & & & $\mathrm{~N}$ & \\
\hline & & $\begin{array}{l}\text { Product Design } \\
\text { Dep. }\end{array}$ & & & & $\mathrm{x}$ & & Ok & \\
\hline \multirow[t]{4}{*}{ Purchases } & $\begin{array}{l}\text { Purchase } \\
\text { Order } \\
\text { Preparation }\end{array}$ & $\begin{array}{l}\text { Product Design } \\
\text { Dep. }\end{array}$ & & & $\mathrm{x}$ & & & $N$ & \\
\hline & \multirow{2}{*}{ Receiving } & Quality Dep. & $x$ & & & & & $\mathrm{~N}$ & \\
\hline & & Suppliers & & & & $\mathrm{x}$ & & Ok & \\
\hline & Payment & $\begin{array}{l}\text { Account } \\
\text { Payable Dep. }\end{array}$ & & & & $\mathrm{x}$ & & OK & \\
\hline
\end{tabular}

Figure 3. Collaboration matrix for the Purchases Department.

URL: http:/mc.manuscriptcentral.com/uism Email: janice.sipior@villanova.edu 


\section{Questionnaire organization for each department}

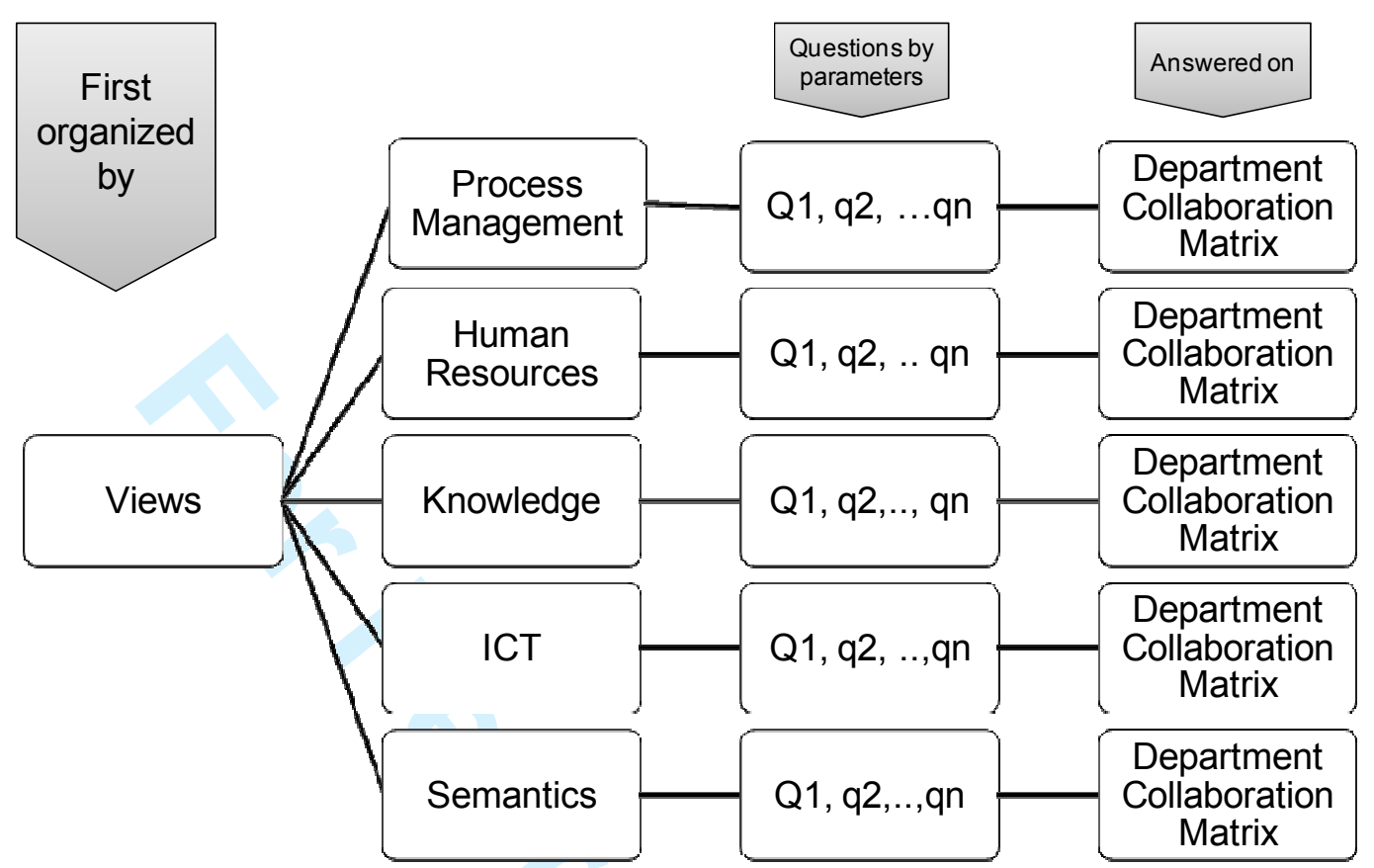

Figure 4. Questionnaire Structure. 


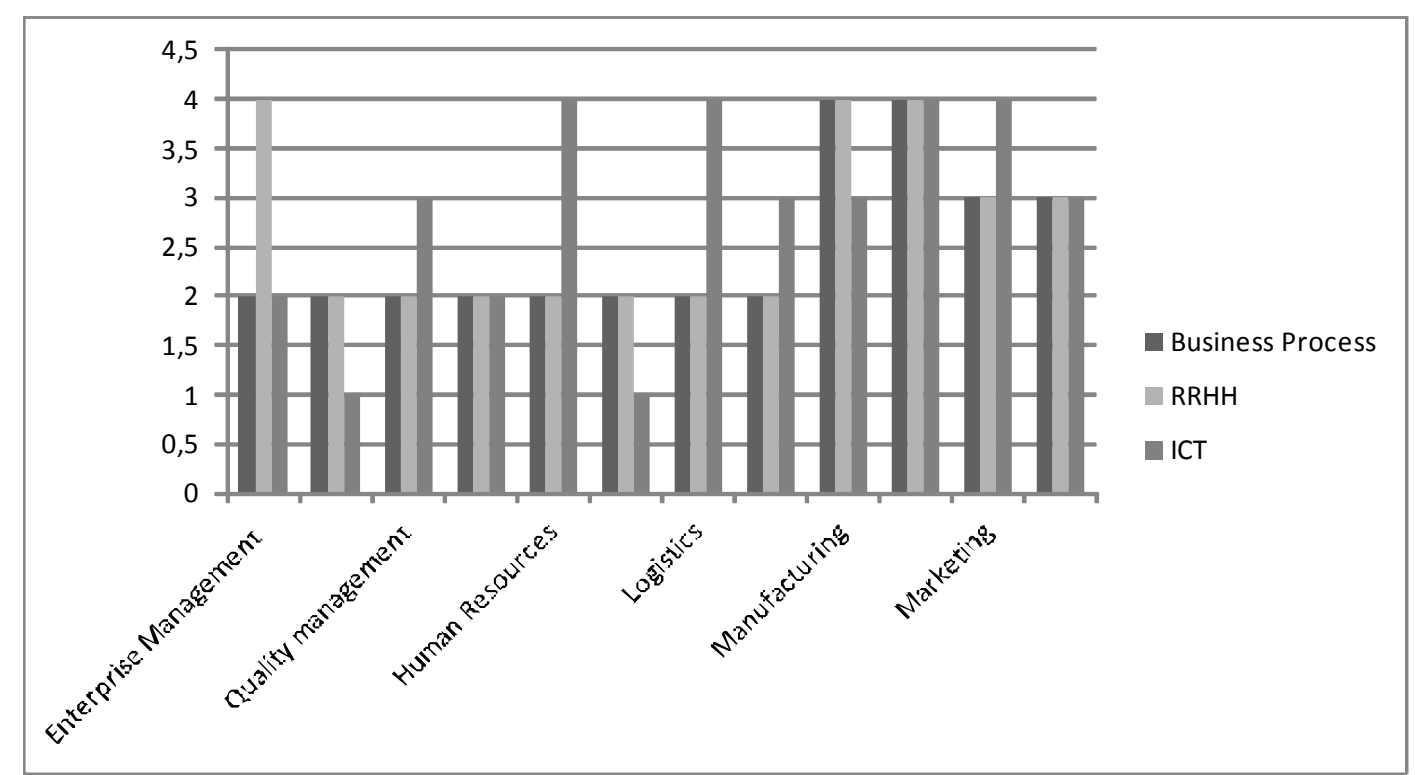

Figure 5. Maturity levels for some departments and views. 